\title{
Ergodic SO(3), monopole condensation and vortex free energy
}

\author{
Giuseppe Burgio* \\ Humboldt-Universität zu Berlin \\ E-mail: burgio@physik.hu-berlin.de

\section{Marcel Fuhrmann} \\ Humboldt-Universität zu Berlin \\ E-mail: fuhrmann@physik.hu-berlin.de \\ Werner Kerler \\ Humboldt-Universität zu Berlin \\ E-mail: kerler@physik.hu-berlin.de \\ Michael Müller-Preussker \\ Humboldt-Universität zu Berlin \\ E-mail: mmp@physik.hu-berlin.de
}

\begin{abstract}
We study the adjoint SU(2) LGT by means of a suppression term for $\mathbb{Z}_{2}$ monopoles. High barriers for tunnelling among different twist sectors are overcome through parallel tempering. Monopole condensation is used to study the finite-temperature phase transition and the properties of the lowtemperature phase. Ergodicity in summing over all twist sectors allows an unbiased measure of the free energy of 't Hooft vortices. Its behaviour in the $\mathrm{SO}(3)$ confined phase hints at differences from what is conjectured for semi-integer discretizations.
\end{abstract}

XXIIIrd International Symposium on Lattice Field Theory

25-30 July 2005

Trinity College, Dublin, Ireland

\footnotetext{
* Speaker.
} 


\section{Introduction}

The spontaneous breaking of global center symmetry [1] observed in simulations of pure fundamental lattice Yang-Mills theories at finite temperature has offered unique insight into their still elusive dynamics [2]. Whether and in what sense this holds for the perturbatively equivalent centerblind adjoint discretization, as universality predicts [㣙, must still be appropriately answered [4]. The difficulties inherent to such non-perturbative regularization have been well known for a long time [5] and are best illustrated by the phase diagram of the mixed action, which for SU(2) reads

$$
S=\beta_{A} \sum_{P}\left(1-\frac{1}{3} \operatorname{Tr}_{A} U_{P}\right)+\beta_{F} \sum_{P}\left(1-\frac{1}{2} \operatorname{Tr}_{F} U_{P}\right) ; \frac{1}{g^{2}}=\frac{1}{4} \beta_{F}+\frac{2}{3} \beta_{A} .
$$

The theory exhibits bulk transitions related to the condensation of $\mathbb{Z}_{2}$ monopoles $\sigma_{c} \in \mathrm{SO}(3)$ and vortices $\sigma_{l} \in \mathrm{SU}(2)$ which hinder the study of its finite temperature properties [6]. First concrete attempts to study it at finite temperature by implementing suppressing chemical potentials $\lambda \sum_{c}(1-$ $\left.\sigma_{c}\right), \gamma \sum_{l}\left(1-\sigma_{l}\right)$, as suggested in [6], were only made relatively recently [7]. For the center blind case $\beta_{F}=0, \gamma=0$, where the bulk transition separates a strong coupling phase I, continuosly connected with SU(2), from a weak coupling phase II, there is no symmetry breaking mechanism and no order parameter. In these works it was however first observed how in such pure adjoint case at high temperature the theory possesses, besides the "regular" deconfined phase, where the adjoint Polyakov loop $L_{A} \rightarrow 1$, a new phase where $L_{A} \rightarrow-1 / 3$. In [8] a dynamical observable measuring the twist expectation value $z$ was introduced after noting that the $\delta\left(\sigma_{c}-1\right)$ constraint effectively implemented by $\mathbb{Z}_{2}$ monopole suppression allows the $\mathrm{SO}(3)$ partition function to be rewritten as the sum of $\mathrm{SU}(2)$ partition functions with all possible twisted boundary conditions $\left.Z\right|_{z=i}$ [9]. The $L_{A} \rightarrow-1 / 3$ phase was linked to a non-trivial twist expectation value, i.e. to the creation of a vortex in the vacuum. The $\mathrm{SO}(3)$ theory was proposed as the ideal test case to check the 't Hooft vortex confinement criterion [10]. Unfortunately twist sectors freeze at the bulk and attempts with a multicanonical algorithm at $\lambda=0$ have been limited to volumes not higher then $8^{3} \times 4$ [8]. Other attempts to study the existence of a finite temperature transition in phase II with $\lambda \neq 0$ through thermodynamic observables were limited to very small temporal extent $N_{\tau}=2$ [11], confirming that the continuum limit is a real challenge for the adjoint theory. A step forward was made in [12, 13], where by means of the Pisa disorder parameter for monopole condensation lines of second order transition properly scaling with $N_{\tau}$ and ending on the bulk where actually found at each fixed twist, with critical exponents consistent with Ising 3-d. Whether such is the case also for the theory summed over all twist sectors and how the vortex free energy behaves in the ergodic simulations is the subject of the present preliminary report, based on the poster presented at this conference. A complete analysis of the model with updated results, including a detailed description of the algorithm and error analysis can be found in [14].

\section{Action and Observables}

As anticipated, we will concentrate on the pure adjoint Wilson action (Eq. (1.1) with $\beta_{F}=$ 0 ), modified by the suppression term $\lambda \sum_{c}\left(1-\sigma_{c}\right)$, where $\sigma_{c}=\prod_{P \in \partial c} \operatorname{sign}\left(\operatorname{Tr}_{F} U_{P}\right)$ around all elementary 3-cubes $c$ defines the $\mathbb{Z}_{2}$ magnetic charge. Its density $M=1-\left\langle\frac{1}{N_{c}} \sum_{c} \sigma_{c}\right\rangle$ tends to 
one in the strong coupling region (phase I) and to zero in the weak coupling limit (phase II), $N_{c}$ denoting the total number of elementary 3 -cubes. Such action is center-blind in the entire $\beta_{A}-\lambda$ plane [15]. We will employ parallel tempering to obtain ergodicity among different twist sectors when evaluating the expectation values of physical observables, e.g. the Pisa disorder parameter and the 't Hooft vortex free energy.

\subsection{Vortex Free Energy}

Temporal twists, corresponding to maximal 't Hooft loop and defining our center vortices, are topological excitations which offer a natural link between center symmetry breaking and degrees of freedom independent of the discretization used [10]. It has been known for a long time in the literature that $\mathrm{SO}(3)$ with $\mathbb{Z}_{2}$ monopole chemical potential in phase II is equivalent to $\mathrm{SU}(2)$ including all possible twisted b.c. [9]

$$
\sum_{\text {b.c. }} Z_{S U(2)}=\left.\int(D U) e^{-S_{S O(3)}} \prod_{c} \delta\left(\sigma_{c}-1\right) \simeq Z_{S O(3)}\right|_{\lambda \rightarrow \infty} .
$$

Above the bulk $\mathrm{SO}(3)$ trades thus boundary conditions with twist sectors. Twists, i.e. 't Hooft loops, thus become observables rather than boundary constraints like in $\mathrm{SU}(2)$ :

$$
z=\frac{1}{N_{s}^{2}} \sum_{j, k \neq i} \prod_{x \in(i, t) \text { plane }} \operatorname{sign}\left(\operatorname{Tr}_{f} U_{i, t}(x)\right) .
$$

Since creating such 't Hooft loop amounts to a change in the signs of some plaquettes, the free energy change $\Delta F=\Delta U-T \Delta S$ will only receive an entropy contribution for adjoint discretizations, the action remaining unmodified in the process. Defining thus the 't Hooft vortex free energy as the ratio of the partition function in the non-trivial twist sector to that in the trivial one $F=$ $-\left.T \log Z\right|_{z=1} /\left.Z\right|_{z=0}$, their relative weight can be measured through an ergodic simulation. To be an order parameter in the thermodynamic limit $\left(V=N_{s}^{3} \rightarrow \infty\right), F$ should vanish exponentially in the confined phase while diverging with an area law $F \sim \tilde{\sigma} N_{s}^{2}$ above the deconfinement transition, where $\tilde{\sigma}$ is the dual string tension. Working at $\lambda=0$ proved however to be a hurdle, since the "freezing" of twist sectors above the bulk transition yields high potential barriers hard to overcome even with a multi-canonical algorithm [8]. From the results in [15] it is sound to conjecture that the whole physically relevant $\mathrm{SO}(3)$ dynamics lies in phase II, the finite temperature transition eventually decoupling from the bulk even at $\lambda=0$. Unfortunately, from estimates in [8], this should not happen for volumes smaller than $\sim 500 \times 1000^{3}$. A non-vanishing $\lambda$ seems therefore the only feasible way to gain access to the properties of the continuum limit of $\operatorname{SO}(3)$. The observation made in [河] that the bulk transition weakens to $2^{\text {nd }}$ order with increasing $\lambda$ will prove crucial in this respect.

\subsection{Pisa disorder parameter}

The Pisa disorder operator, measuring monopole condensation, is an order parameter for the dual superconductor mechanism of confinement [16]: It is defined through $S_{M}$, the action modified through a bosonic field $\Phi$ introduced at fixed time and invariant under a residual gauge symmetry $\mathrm{U}(1)$, does not dependent from the particular choice for $\Phi$ and is well defined, independently of 
center symmetry, also for full QCD. Its derivative, $\rho$, is easier to compute in actual numerical simulations:

$$
\langle\mu\rangle=\frac{\int(D U) e^{-\left(S_{M}-S\right)} e^{-S}}{\int(D U) e^{-S}}=\exp \left(\int_{0}^{\beta} \rho\left(\beta^{\prime}\right) d \beta^{\prime}\right) .
$$

For $T<T_{c}\langle\mu\rangle \neq 0$ signals spontaneous breaking of $\mathrm{U}(1)$, corresponding to $\rho \sim 0$, bounded from below for $N_{s} \rightarrow \infty$. At the phase transition $\rho$ should show a sharp negative peak at $\beta^{c}$ diverging for $N_{s} \rightarrow \infty$, while for $T>T_{c}\langle\mu\rangle=0$ corresponds to the trivial vacuum $\left(\rho \propto-N_{s}\right.$ for $\left.N_{s} \rightarrow \infty\right)$. More details regarding its implementation for $\mathrm{SO}(3)$ can be found in [12, 13].

\section{Fixed twist dynamics}

It was shown in [13] that for $\mathrm{SO}$ (3) in phase II at fixed twist and low $\beta_{A} \rho \sim 0 \forall z$, as one would expect from a confined phase; $\rho$ peaks at some ( $z$ dependent!) $\beta_{A}^{c}$; at high $\beta_{A} \rho$ diverges for $z=0$, while it vanishes for $z \neq 0$. Moreover each $z$-sector can be mapped in a different positive plaquette model, making fundamental observables measurable while the adjoint sector remains untouched. This is consistent with different twist sectors possessing slightly different underlying fundamental dynamics in the confined phase, but very different ones above deconfinement, the twist behaving effectively like a background field. Each twist sector measures slightly different string tensions in the confined phase (through Creutz ratios and Polyakov loop correlators) and deconfinement temperatures [13]. From the above fixed twist measurements it is thus natural to expect that the ergodic expectation value of $\mu$

$$
\langle\mu\rangle=\frac{\left.\left.\sum_{i} \mu\right|_{z=i} Z_{S O(3)}\right|_{z=i}}{\left.\sum_{i} Z_{S O(3)}\right|_{z=i}}
$$

at low $\beta_{A}$ will be $\langle\mu\rangle \simeq 0$, while at high $\left.\beta_{A}\langle\mu\rangle \simeq\langle\mu\rangle\right|_{z=0}\left(1-e^{-F / T}\right)$. In whole phase II the ergodic theory would be consistent with confinement at low $\beta_{A}$ and with deconfinement at high $\beta_{A}$. To establish whether $\mu$ actually still behaves as an order parameter its expectation value at the physical transition must be studied through an ergodic algorithm.

\section{Parallel Tempering}

The idea of Parallel tempering (see [17] for a review) is to simulate at the same time several ensembles above and below the bulk transition. Each ensemble is caracterized by a link-configuration $C_{i}$ and a set of couplings $\beta_{i}$. To reach ergodicity one lets them evolve separately swapping every few MC steps the $i^{t h}$ and $(i-1)^{t h}$ ensemble through a generalized Metropolis step with weight

$$
W_{i}=e^{-\left(S\left(\beta_{i}, C_{i-1}\right)+S\left(\beta_{i-1}, C_{i}\right)\right)+\left(S\left(\beta_{i}, C_{i}\right)+S\left(\beta_{i-1}, C_{i-1}\right)\right)} .
$$

As Fig. 1 shows this works very well, although it is crucial to fine tune the parameters to optimize acceptance rate and keep auto- and cross-correlations under control [14]. Since phase I has a high tunnelling probability, the softening of the bulk transition to $2^{\text {nd }}$ order is crucial to "transport" tunneling into phase II. Working on the $1^{\text {st }}$ order bulk branch would have led to high barriers and thus to no ergodicity for large volume. 

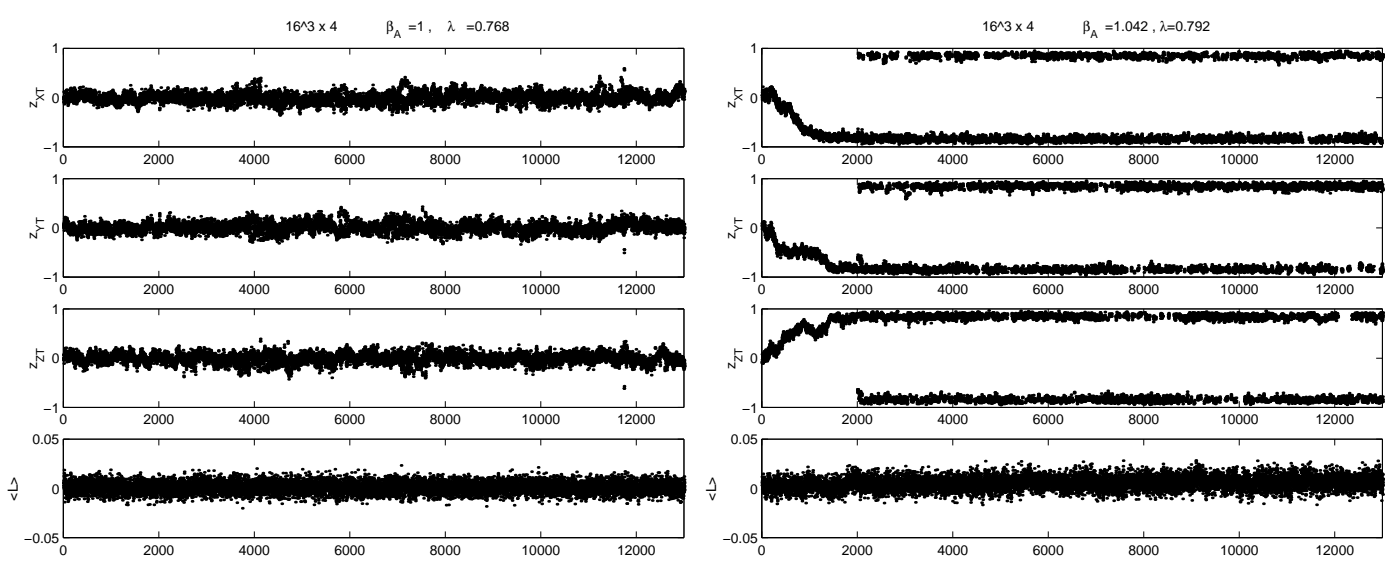

Figure 1: $\mathrm{MC}$ history of the twist variables and the adjoint Polyakov loop $L_{A}$ below the bulk transition for $\left(\beta_{A}, \lambda\right)=(1 ., 0.768)$ (left) and above the transition $(1.042,0.792)$ (right) belonging to the same parallel tempering run $\left(V=16^{3} \times 4\right)$.

\section{Results}

As explained in Sect. 3, we need to establish whether $\rho$ gives a clear signal for a phase transition through ergodic runs. This is shown in Fig. 2-left. Due to the algorithm, we must choose a path that starts close to the bulk and goes above the deconfinement transition, with both branches not too far away from each other. Such path gives competing effects, since $\rho$ also diverges at the bulk [12, 13]. As for $F$, in phase I and at high $\beta_{A}$ it behaves as expected. The surprise comes in the confined phase of phase II, where the free energy doesn't vanish (Fig. 2-right), taking negative values. We checked that away from both transitions $F$ also reaches constant, $\beta_{A}$ dependent negative values throughout the confined phase II.
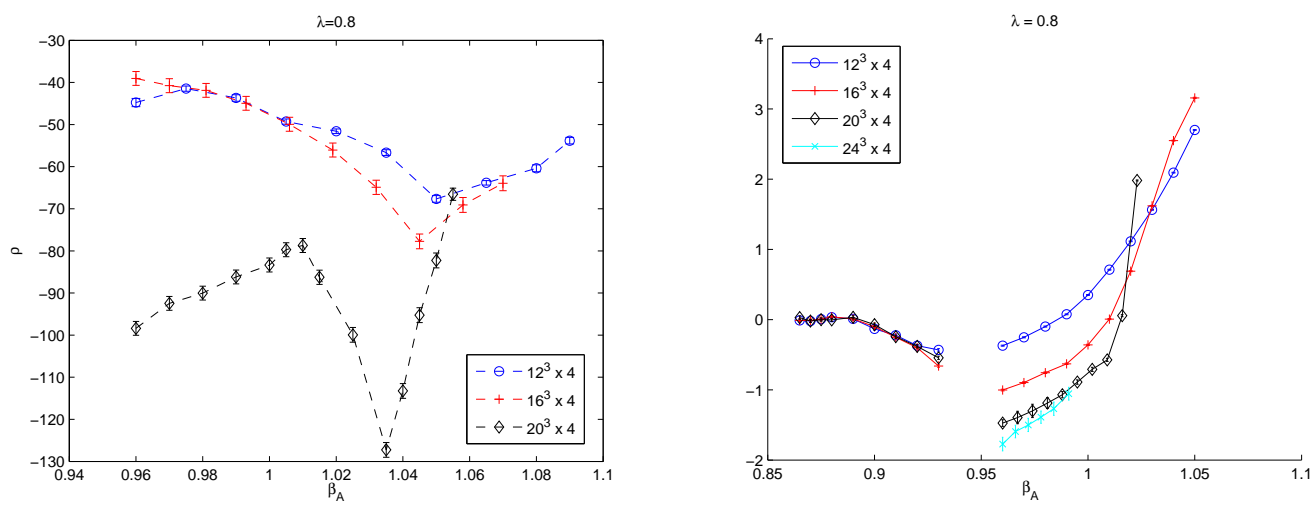

Figure 2: Pisa disorder operator (left) and vortex free energy (right).

\section{Conclusion and Outlook}

We study the finite temperature transition in a modified $\mathrm{SO}(3)$ lattice gauge theory. Sum over 
twists for ergodicity is reached through parallel tempering. The Pisa disorder parameter is used to characterize the properties of the different phases, so that monopole (de)condensation could be used to obtain the critical exponents. Although well defined for $\mathrm{SO}(3)$, the 't Hooft vortex free energy behaves unexpectedly, being negative in the confined phase of $\mathrm{SO}(3)$. The meaning of such vortex enhancement could be relevant for the dynamics of continuum Yang-Mills theories. However, contrary to $\mu, F$ seems not to behave as an order parameter in the theory discretized without fundamental components. Keeping in mind the full QCD case, there actually seems to be no compelling reason why in a center-blind regularization of Yang-Mills theory an order parameter for center symmetry breaking should vanish. Universal, representation independent observables should however be preserved, so further confirmations of the correct physical behaviour of the adjoint dynamics through e.g. the glueball spectrum might therefore be interesting. Also, the extension to SU(3) should shed further light on the problem. For further discussions and a more detailed analysis we refer to [14].

\section{References}

[1] A. M. Polyakov, Phys. Lett. B72 (1978) 477-480; L. Susskind, Phys. Rev. D20 (1979) 2610-2618.

[2] L. D. McLerran, B. Svetitsky, Phys. Lett. B98 (1981) 195; J. Kuti et al. , Phys. Lett. B98 (1981) 199.

[3] B. Svetitsky, L. G. Yaffe, Nucl. Phys. B210 (1982) 423.

[4] A. V. Smilga, Ann. Phys. 234 (1994) 1-59.

[5] G. Bhanot, M. Creutz, Phys.Rev.D24 (1981) 3212.; J. Greensite, B. Lautrup, Phys. Rev. Lett.47 (1981) 9.

[6] I. G. Halliday, A. Schwimmer, Phys. Lett. B101 (1981) 327; Phys. Lett. B102 (1981) 337.

[7] S. Datta, R. V. Gavai, Phys. Rev. D57 (1998) 6618-6624.

[8] P. de Forcrand, O. Jahn, Nucl. Phys. B651 (2003) 125-142.

[9] G. Mack, V. B. Petkova, Zeit. Phys. C12 (1982) 177; E. Tomboulis, Phys. Rev. D23 (1981) 2371.

[10] G. 't Hooft, Nucl. Phys. B138 (1978) 1; Nucl. Phys. B153 (1979) 141.

[11] S. Datta, R. V. Gavai, Nucl. Phys. Proc. Suppl. 83 (2000) 366-368; Phys. Rev. D60 (1999) 034505.

[12] A. Barresi, G. Burgio, M. D’Elia, M. Müller-Preussker, Phys. Lett. B599 (2004) 278-284.

[13] A. Barresi, G. Burgio, in preparation.

[14] G. Burgio, M. Fuhrmann, W. Kerler, M. Müller-Preussker, in preparation

[15] A. Barresi, G. Burgio, M. Müller-Preussker, Phys. Rev. D69 (2004) 094503.

[16] A. Di Giacomo, G. Paffuti, Phys. Rev. D56 (1997) 6816-6823; A. Di Giacomo, B. Lucini, L. Montesi, G. Paffuti, Phys. Rev. D61 (2000) 034503.

[17] E. Marinari, cond-mat/9612010. 